\title{
Nonexistence of solutions for the quasilinear parabolic differential inequalities with singular potential term and nonlocal source
}

\author{
Suping Xiao ${ }^{1}$ and Zhong Bo Fang ${ }^{2^{*}}$
}

\section{"Correspondence:}

fangzb7777@hotmail.com

${ }^{2}$ School of Mathematical Sciences,

Ocean University of China, Qingdao,

P.R. China

Full list of author information is

available at the end of the article

\section{Springer}

\begin{abstract}
This paper is devoted to proving some new nonexistence theorems for a class of quasilinear parabolic differential inequalities with a singular potential term and nonlocal source term in the case of homogeneous and non-homogeneous by the test function method.
\end{abstract}

MSC: 35K59; 35K15; 35B53

Keywords: Singular potential term; Nonlocal source; Test function; Nonexistence

\section{Introduction}

In this paper, we consider the homogeneous and non-homogeneous inequalities with singular potential and weight nonlocal source term of the form

$$
\begin{aligned}
& u_{t} \geq \Delta u^{m}-V(x) u+|x|^{\alpha} u^{p}\left\|\beta^{\frac{1}{q}}(x) u\right\|_{q}^{r}, \quad(x, t) \in R^{N} \times(0, \infty), \\
& u_{t} \geq \Delta u^{m}-V(x) u+|x|^{\alpha} u^{p}\left\|\beta^{\frac{1}{q}}(x) u\right\|_{q}^{r}+\omega(x), \quad(x, t) \in R^{N} \times(0, \infty),
\end{aligned}
$$

respectively, subject to the initial condition

$$
u(x, 0)=u_{0}(x), \quad x \in R^{N},
$$

where $m, p, q, r, \alpha>0, q(p+r)>\max \{1, m\}(q+r)$, the initial function $u_{0}(x) \in L_{\mathrm{loc}}^{1}\left(R^{N}\right)$ is nonnegative, the singular potential $V(x)$ is a positive function satisfying $V(x) \sim|x|^{-\sigma}$, $\sigma>0$, and the weight function $\beta(x)$ is positive and singular at the origin, i.e., there exist constants $c>0$ and $s \in R_{+}$such that

$$
\beta(x) \geq c|x|^{-s}>0, \quad x \in R^{N} \backslash\{0\},
$$

and the non-homogeneous term $\omega(x) \geq 0$ and satisfy $\omega(x) \in L_{\text {loc }}^{1}\left(R^{N}\right)$.

Our model (1.1) describes the diffusion of concentration of some Newtonian fluids through a porous medium or the density of some biological species in many physical phenomena and biological species theories. However, it is well known that the nonlocal source

(c) The Author(s) 2020. This article is licensed under a Creative Commons Attribution 4.0 International License, which permits use, sharing, adaptation, distribution and reproduction in any medium or format, as long as you give appropriate credit to the original author(s) and the source, provide a link to the Creative Commons licence, and indicate if changes were made. The images or other third party material in this article are included in the article's Creative Commons licence, unless indicated otherwise in a credit line to the material. If material is not included in the article's Creative Commons licence and your intended use is not permitted by statutory regulation or exceeds the permitted use, you will need to obtain permission directly from the copyright holder. To view a copy of this licence, visit http://creativecommons.org/licenses/by/4.0/. 
term presents a more realistic model for population dynamics, see [1-3]. In the nonlinear diffusion theory, there exist obvious differences among the situations of slow $(m>1)$, fast $(m<1)$, and linear $(m=1)$ diffusions. For example, there is a finite speed propagation in the slow and linear diffusion situations, whereas an infinite speed propagation exists in the fast diffusion situation.

In 1966, Fujita [4] studied the following Cauchy problem of the semilinear heat equation with a local source term:

$$
u_{t}=\Delta u+u^{p}, \quad(x, t) \in R^{N} \times(0, \infty)
$$

and obtained the critical exponent $q_{c}=1+\frac{2}{N}$ on the existence versus nonexistence of nonnegative nontrivial global solution. Later, the study of global existence and nonglobal existence of solutions for nonlinear parabolic type partial differential equations (or inequalities) also made some progress. The nonexistence of global solutions is a nonlinear Liouville type theorem. Thus one can prove some properties of solutions in a bounded domain which is a kind of essential reflection of blow-up or singularity theory as well, see [5] and the references therein.

As far as we know, there have been many results about nonexistence of global solutions for Cauchy problems of local parabolic type differential equations (or inequalities) without potential term, we refer the readers to [6-9] (quasilinear elliptic or parabolic equations without singular variable coefficient), [10-13] (quasilinear elliptic or parabolic equations with singular variable coefficient), and the references therein.

For the studies on the local quasilinear parabolic equation (or inequality) with a potential term, Yang et al. [14] considered the Cauchy problem of the fast diffusion equation with a potential term of the form

$$
u_{t}=\Delta u^{m}-V(x) u^{m}+u^{p}, \quad(x, t) \in R^{N} \times(0, T) .
$$

They obtained the critical Fujita exponent $p_{c}=m+\frac{2}{m \alpha+n}$ to the problem in the sense that every nontrivial solution blows up in finite time if $1<p \leq p_{c}$ and there are both global and nonglobal solutions if $p>p_{c}$ by using the test function method and constructing a supersolution technique. Liu [15] also studied the above problem in a conical domain. For more results on the critical Fujita exponent of semilinear heat equations with the potential term, one can refer to $[16,17]$.

For the studies on nonlocal problem, Chen et al. [18] considered some degenerated parabolic inequalities with the local and nonlocal nonlinear term

$$
\frac{\partial u^{m}}{\partial t} \geq \Delta u+\|u(t)\|_{p}^{p}+b(x, t) u^{q}(x, t), \quad(x, t) \in R^{N} \times(0, \infty),
$$

and

$$
\frac{\partial u^{m}}{\partial t} \geq \Delta u+\left(\int_{R^{n}} \beta(y) u^{p}(y, t) d y\right)^{\frac{n}{p}} u^{q}(x, t), \quad(x, t) \in R^{N} \times(0, \infty) .
$$

They obtained the global nonexistence of nontrivial solutions by the test function method. Later, Huang et al. [19] studied the global nonexistence of solutions to a time degenerate type evolution problems with nonlocal sources. 
Considering the above works, one can find that the study on the nonexistence of solutions for the homogeneous and non-homogeneous quasilinear differential inequalities with a singular potential term and a weighted nonlocal source term has not been started yet. The purpose of this paper is to establish the nonexistence of solutions for homogeneous and non-homogeneous Cauchy problems by the test function method $[6,7,13]$. For this, there are two difficult points. One point is to find the influence of singular potential term, weighted function, nonlocal source term, homogeneous and non-homogenous terms on the nonexistence of a nonnegative nontrivial global weak solution. The other one is the choice of a suitable test function. Particularly, the comparison results and the conditions at infinity for the solutions are not required.

The rest of our paper is organized as follows. In Sect. 2, we introduce some preliminaries including some definitions and the main results. The proofs of the main results are given in Sect. 3.

\section{Preliminaries and main results}

Firstly, we give some definitions about a weak solution.

Definition 1 A nonnegative function $u(x, t)$ is called a weak solution of problem (1.1), (1.3) if the following conditions hold:

(i) $u, u^{m}, V(x) u,|x|^{\alpha} u^{p}\left\|\beta^{\frac{1}{q}}(x) u\right\|_{q}^{r} \in L_{\mathrm{loc}}^{1}\left(R^{N} \times(0, \infty)\right)$;

(ii) for any nonnegative function $\zeta \in C_{0}^{2,1}\left(R^{N} \times(0, \infty)\right)$, we have

$$
\begin{aligned}
& \int_{0}^{\infty} \int_{R^{N}}|x|^{\alpha} u^{p} \zeta\left\|\beta^{\frac{1}{q}}(x) u\right\|_{q}^{r} d x d t+\int_{R^{N}} u_{0}(x) \zeta(x, 0) d x \\
& \leq-\int_{0}^{\infty} \int_{R^{N}}\left(u \zeta_{t}+u^{m} \triangle \zeta\right) d x d t+\int_{0}^{\infty} \int_{R^{N}} V(x) u \zeta d x d t
\end{aligned}
$$

Similarly, a nonnegative function $u(x, t)$ is called a weak solution of non-homogeneous problem (1.2), (1.3) if the following inequality holds:

$$
\begin{aligned}
& \int_{0}^{\infty} \int_{R^{N}}|x|^{\alpha} u^{p} \zeta\left\|\beta^{\frac{1}{q}}(x) u\right\|_{q}^{r} d x d t+\int_{R^{N}} u_{0}(x) \zeta(x, 0) d x+\int_{0}^{\infty} \int_{R^{N}} \omega(x) \zeta d x d t \\
& \leq-\int_{0}^{\infty} \int_{R^{N}}\left(u \zeta_{t}+u^{m} \triangle \zeta\right) d x d t+\int_{0}^{\infty} \int_{R^{N}} V(x) u \zeta d x d t .
\end{aligned}
$$

Definition 2 Suppose that a nonnegative function $u(x, t)$ is a weak solution of problem (1.1), (1.3) in $S=R^{N} \times(0, \infty)$, then $u(x, t)$ is said to be a solution with positive lower bound in $S$ if there exists $c>0$ such that $u(x, t) \geq c>0$ a.e. in $S$.

Secondly, we show main results as follows.

Theorem 1 If $\alpha>\frac{r s}{q}$ and $1 \leq N \leq \frac{2 q+\alpha q-r s}{q(r+p-m)-r}$, then homogeneous problem (1.1), (1.3) has no nontrivial global solution.

Theorem 2 Suppose $s<N+\frac{q(2+\alpha)}{r}$, then homogeneous problem (1.1), (1.3) has no solution with positive lower bound in $S$.

Theorem 3 If the initial data $u_{0}$ is compactly supported, and 
(i) either $N \leq 2$ or

(ii) $N>2, N<\frac{2 q(p+r)+m(\alpha q-r s)}{q(p+r)-m(q+r)}$,

then non-homogeneous problem (1.2), (1.3) has no nontrivial global solution.

\section{The proof of main results}

Proof of Theorem 1 We let $u(x, t)$ be a weak solution of homogeneous problem (1.1), (1.3) and $\zeta$ is a nonnegative smooth test function, then we know

$$
\begin{aligned}
& \int_{0}^{\infty} \int_{R^{N}}|x|^{\alpha} u^{p} \zeta\left\|\beta^{\frac{1}{q}}(x) u\right\|_{q}^{r} d x d t+\int_{R^{N}} u_{0}(x) \zeta(x, 0) d x \\
& \quad \leq \int_{0}^{\infty} \int_{R^{N}} u|\zeta t| d x d t+\int_{0}^{\infty} \int_{R^{N}} u^{m}|\triangle \zeta| d x d t+\int_{0}^{\infty} \int_{R^{N}} V(x) u \zeta d x d t
\end{aligned}
$$

Now, we estimate the right-hand side of inequality (3.1). First, applying the Hölder inequality to the first term in the right-hand side of (3.1), we get

$$
\begin{aligned}
\int_{R^{N}} u\left|\zeta_{t}\right| d x \leq & \left(\int_{R^{N}}|x|^{\alpha} u^{p} \zeta d x\right)^{\frac{1}{\alpha_{1}}}\left(\int_{R^{N}} \beta(x) u^{q} d x\right)^{\frac{r}{q \alpha_{1}}} \\
& \times\left(\int_{R^{N}} \beta^{-\frac{r \alpha_{2}}{q \alpha_{1}}}(x)|x|^{-\frac{\alpha \alpha_{2}}{\alpha_{1}}}\left|\zeta_{t}\right|^{\alpha_{2}} \zeta^{-\frac{\alpha_{2}}{\alpha_{1}}} d x\right)^{\frac{1}{\alpha_{2}}} .
\end{aligned}
$$

By the Hölder and Yöung inequalities again, we obtain

$$
\begin{aligned}
\int_{0}^{\infty} & \int_{R^{N}} u\left|\zeta_{t}\right| d x d t \\
\leq & \int_{0}^{\infty}\left(\int_{R^{N}}|x|^{\alpha} u^{p} \zeta d x\right)^{\frac{1}{\alpha_{1}}}\left(\int_{R^{N}} \beta(x) u^{q} d x\right)^{\frac{r}{q \alpha_{1}}} \\
& \times\left(\int_{R^{N}} \beta^{-\frac{r \alpha_{2}}{q \alpha_{1}}}(x)|x|^{-\frac{\alpha \alpha_{2}}{\alpha_{1}}}\left|\zeta_{t}\right|^{\alpha_{2}} \zeta^{-\frac{\alpha_{2}}{\alpha_{1}}} d x\right)^{\frac{1}{\alpha_{2}}} d t \\
\leq & \left(\int_{0}^{\infty} \int_{R^{N}}|x|^{\alpha} u^{p} \zeta\left\|\beta^{\frac{1}{q}}(x) u\right\|_{q}^{r} d x d t\right)^{\frac{1}{\alpha_{1}}} \\
& \times\left(\int_{0}^{\infty}\left(\int_{R^{N}} \beta^{-\frac{r \alpha_{2}}{q \alpha_{1}}}(x)|x|^{-\frac{\alpha \alpha_{2}}{\alpha_{1}}}\left|\zeta_{t}\right|^{\alpha_{2}} \zeta^{-\frac{\alpha_{2}}{\alpha_{1}}} d x\right)^{\frac{\alpha_{1}^{\prime}}{\alpha_{2}}} d t\right)^{\frac{1}{\alpha_{1}^{\prime}}} \\
\leq & \frac{1}{4} \int_{0}^{\infty} \int_{R^{N}}|x|^{\alpha} u^{p} \zeta\left\|\beta^{\frac{1}{q}}(x) u\right\|_{q}^{r} d x d t \\
& +C \int_{0}^{\infty}\left(\int_{R^{N}} \beta^{-\frac{r \alpha_{2}}{q \alpha_{1}}}(x)|x|^{-\frac{\alpha \alpha_{2}}{\alpha_{1}}}\left|\zeta_{t}\right|^{\alpha_{2}} \zeta^{-\frac{\alpha_{2}}{\alpha_{1}}} d x\right)^{\frac{\alpha_{1}^{\prime}}{\alpha_{2}}} d t
\end{aligned}
$$

where

$$
\alpha_{1}=p+r>1, \quad \alpha_{2}=\frac{q(p+r)}{q(p+r)-(q+r)}>1, \quad \frac{1}{\alpha_{1}^{\prime}}=1-\frac{1}{\alpha_{1}} .
$$


Applying similar arguments like the first term of (3.1) to the second and third terms, we have

$$
\begin{aligned}
\int_{0}^{\infty} \int_{R^{N}} u^{m}|\triangle \zeta| d x d t \leq & \frac{1}{4} \int_{0}^{\infty} \int_{R^{N}}|x|^{\alpha} u^{p} \zeta\left\|\beta^{\frac{1}{q}}(x) u\right\|_{q}^{r} d x d t \\
& +C \int_{0}^{\infty}\left(\int_{R^{N}} \beta^{-\frac{r \alpha_{4}}{q_{3}}}(x)|x|^{-\frac{\alpha \alpha_{4}}{\alpha_{3}}}|\Delta \zeta|^{\alpha_{4}} \zeta^{-\frac{\alpha_{4}}{\alpha_{3}}} d x\right)^{\frac{\alpha_{3}^{\prime}}{\alpha_{4}}} d t
\end{aligned}
$$

and

$$
\begin{aligned}
\int_{0}^{\infty} \int_{R^{N}} V(x) u \zeta d x d t \leq & \frac{1}{4} \int_{0}^{\infty} \int_{R^{N}}|x|^{\alpha} u^{p} \zeta\left\|\beta^{\frac{1}{q}}(x) u\right\|_{q}^{r} d x d t \\
& +C \int_{0}^{\infty}\left(\int_{R^{N}} \beta^{-\frac{r \alpha_{2}}{q \alpha_{1}}}(x)|x|^{\left(-\sigma-\frac{\alpha}{\alpha_{1}}\right) \alpha_{2}} \zeta^{\frac{\alpha_{2}}{\alpha_{1}^{\gamma}}} d x\right)^{\frac{\alpha_{1}^{\prime}}{\alpha_{2}}} d t
\end{aligned}
$$

where

$$
\alpha_{3}=\frac{p+r}{m}>1, \quad \alpha_{4}=\frac{q(p+r)}{q(p+r)-m(q+r)}>1, \quad \frac{1}{\alpha_{3}^{\prime}}=1-\frac{1}{\alpha_{3}} .
$$

By (3.1)-(3.4), we obtain

$$
\begin{aligned}
\int_{0}^{\infty} & \int_{R^{N}}|x|^{\alpha} u^{p} \zeta\left\|\beta^{\frac{1}{q}}(x) u\right\|_{q}^{r} d x d t+4 \int_{R^{N}} u_{0}(x) \zeta(x, 0) d x \\
\leq & C \int_{0}^{\infty}\left(\int_{R^{N}} \beta^{-\frac{r \alpha_{2}}{q \alpha_{1}}}(x)|x|^{-\frac{\alpha \alpha_{2}}{\alpha_{1}}}\left|\zeta_{t}\right|^{\alpha_{2}} \zeta^{-\frac{\alpha_{2}}{\alpha_{1}}} d x\right)^{\frac{\alpha_{1}^{\prime}}{\alpha_{2}}} d t \\
& +C \int_{0}^{\infty}\left(\int_{R^{N}} \beta^{-\frac{r \alpha_{2}}{q \alpha_{1}}}(x)|x|^{\left(-\sigma-\frac{\alpha}{\alpha_{1}}\right) \alpha_{2}} \zeta^{\frac{\alpha_{2}}{\alpha_{1}^{\prime}}} d x\right)^{\frac{\alpha_{1}^{\prime}}{\alpha_{2}}} d t \\
& +C \int_{0}^{\infty}\left(\int_{R^{N}} \beta^{-\frac{r \alpha_{4}}{q \alpha_{3}}}(x)|x|^{-\frac{\alpha \alpha_{4}}{\alpha_{3}}}|\triangle \zeta|^{\alpha_{4}} \zeta^{-\frac{\alpha_{4}}{\alpha_{3}}} d x\right)^{\frac{\alpha_{3}^{\prime}}{\alpha_{4}}} d t .
\end{aligned}
$$

Now, we take $\zeta(x, t)=\varphi\left(\rho^{-\theta} t\right) \varphi\left(\rho^{-2}|x|^{2}\right)$, where $\varphi \in C_{0}^{\infty}\left(R_{+}\right)$satisfies $0 \leq \varphi \leq 1$ and

$$
\varphi(x)= \begin{cases}0, & |x| \geq 2 \\ 1, & 0 \leq|x| \leq 1\end{cases}
$$

where $\rho$ is a positive parameter. In order to estimate the right-hand side of (3.5), we consider the change of variables $x=\rho \xi, t=\rho^{\theta} \tau$, then

$$
\begin{aligned}
& \int_{0}^{\infty}\left(\int_{R^{N}} \beta^{-\frac{r \alpha_{2}}{q \alpha_{1}}}(x)|x|^{-\frac{\alpha \alpha_{2}}{\alpha_{1}}}\left|\zeta_{t}\right|^{\alpha_{2}} \zeta^{-\frac{\alpha_{2}}{\alpha_{1}}} d x\right)^{\frac{\alpha_{1}^{\prime}}{\alpha_{2}}} d t \\
& \leq \rho^{\theta+\left(N-\theta \alpha_{2}+\frac{r s \alpha_{2}}{q \alpha_{1}}-\frac{\alpha \alpha_{2}}{\alpha_{1}}\right) \frac{\alpha_{1}^{\prime}}{\alpha_{2}}} \int_{1}^{2}\left(\int_{|\xi| \leq 2}|\xi|^{\frac{r s \alpha_{2}}{q \alpha_{1}}-\frac{\alpha \alpha_{2}}{\alpha_{1}}} \zeta^{-\frac{\alpha_{2}}{\alpha_{1}}}\left|\zeta_{\tau}\right|^{\alpha_{2}} d \xi\right)^{\frac{\alpha_{1}^{\prime}}{\alpha_{2}}} d \tau \\
& \leq C_{1} \rho^{\theta+\left(N-\theta \alpha_{2}+\frac{r s \alpha_{2}}{q \alpha_{1}}-\frac{\alpha \alpha_{2}}{\alpha_{1}}\right) \frac{\alpha_{1}^{\prime}}{\alpha_{2}}} .
\end{aligned}
$$


Xiao and Fang Journal of Inequalities and Applications

(2020) 2020:64

Page 6 of 9

$$
\int_{0}^{\infty}\left(\int_{R^{N}} \beta^{-\frac{r \alpha_{2}}{q \alpha_{1}}}(x)|x|^{\left(-\sigma-\frac{\alpha}{\alpha_{1}}\right) \alpha_{2}} \zeta^{\frac{\alpha_{2}}{\alpha_{1}^{\prime}}} d x\right)^{\frac{\alpha_{1}^{\prime}}{\alpha_{2}}} d t \leq C_{2} \rho^{\theta+\left(N-\left(\sigma+\frac{\alpha}{\alpha_{1}}\right) \alpha_{2}+\frac{r s \alpha_{2}}{q \alpha_{1}}\right) \frac{\alpha_{1}^{\prime}}{\alpha_{2}}}
$$

and

$$
\int_{0}^{\infty}\left(\int_{R^{N}} \beta^{-\frac{r \alpha_{4}}{q \alpha_{3}}}(x)|x|^{-\frac{\alpha \alpha_{4}}{\alpha_{3}}}|\Delta \zeta|^{\alpha_{4}} \zeta^{-\frac{\alpha_{4}}{\alpha_{3}}} d x\right)^{\frac{\alpha_{3}^{\prime}}{\alpha_{4}}} d t \leq C_{3} \rho^{\theta+\left(N-2 \alpha_{4}+\frac{r s \alpha_{4}}{q \alpha_{3}}-\frac{\alpha \alpha_{4}}{\alpha_{3}}\right) \frac{\alpha_{3}^{\prime}}{\alpha_{4}}}
$$

where $C_{1}, C_{2}$, and $C_{3}$ are constants that are not dependent on $\rho$. From (3.5)-(3.8), we get

$$
\begin{aligned}
& \int_{0}^{\infty} \int_{R^{N}}|x|^{\alpha} u^{p} \zeta\left\|\beta^{\frac{1}{q}}(x) u\right\|_{q}^{r} d x d t+4 \int_{R^{N}} u_{0}(x) \zeta(x, 0) d x \\
& \leq C \rho^{\theta+\left(N-\theta \alpha_{2}+\frac{r s \alpha_{2}}{q \alpha_{1}}-\frac{\alpha \alpha_{2}}{\alpha_{1}}\right) \frac{\alpha_{1}^{\prime}}{\alpha_{2}}}+C \rho^{\theta+\left(N-\left(\sigma+\frac{\alpha}{\alpha_{1}}\right) \alpha_{2}+\frac{r s \alpha_{2}}{q \alpha_{1}}\right) \frac{\alpha_{1}^{\prime}}{\alpha_{2}}} \\
& \quad+C \rho^{\theta+\left(N-2 \alpha_{4}+\frac{r s \alpha_{4}}{q \alpha_{3}}-\frac{\alpha \alpha_{4}}{\alpha_{3}}\right) \frac{\alpha_{3}^{\prime}}{\alpha_{4}}} .
\end{aligned}
$$

We choose $\theta$ such that

$$
\begin{aligned}
\theta+\left(N-\theta \alpha_{2}+\frac{r s \alpha_{2}}{q \alpha_{1}}-\frac{\alpha \alpha_{2}}{\alpha_{1}}\right) \frac{\alpha_{1}^{\prime}}{\alpha_{2}} & =\theta+\left(N-\left(\sigma+\frac{\alpha}{\alpha_{1}}\right) \alpha_{2}+\frac{r s \alpha_{2}}{q \alpha_{1}}\right) \frac{\alpha_{1}^{\prime}}{\alpha_{2}} \\
& =\theta+\left(N-2 \alpha_{4}+\frac{r s \alpha_{4}}{q \alpha_{3}}-\frac{\alpha \alpha_{4}}{\alpha_{3}}\right) \frac{\alpha_{3}^{\prime}}{\alpha_{4}}
\end{aligned}
$$

i.e.,

$$
\theta=\sigma=\left(\frac{r s}{q}-\alpha\right) \frac{1-m}{p+r-m}+N \frac{(m-1) r}{q(p+r-m)}+2 \frac{p+r-1}{p+r-m} .
$$

Such a choice gives a common value $\gamma$ of the exponents of $\rho$ in (3.9), namely

$$
\gamma=\frac{1}{p+r-m}\left(\left(\frac{r s}{q}-\alpha\right)+N\left(\frac{q(p+r-m)-r}{q}\right)-2\right) .
$$

If $\gamma<0$, the right-hand side of (3.9) goes to 0 when $\rho$ goes to infinity, clearly this implies that $u$ cannot exist.

If $\gamma=0$, then $\int_{0}^{\infty} \int_{R^{N}}|x|^{\alpha} u^{p} \zeta\left\|\beta^{\frac{1}{q}}(x) u\right\|_{q}^{r} d x d t<\infty$. We return to inequality (3.1), which actually reads

$$
\begin{aligned}
\int_{0}^{\infty} & \int_{R^{N}}|x|^{\alpha} u^{p} \zeta\left\|\beta^{\frac{1}{q}}(x) u\right\|_{q}^{r} d x d t+\int_{R^{N}} u_{0}(x) \zeta(x, 0) d x \\
\leq & \int_{0}^{\infty} \int_{R^{N}} u\left|\zeta_{t}\right| d x d t+\int_{0}^{\infty} \int_{R^{N}} u^{m}|\triangle \zeta| d x d t+\int_{0}^{\infty} \int_{R^{N}} V(x) u \zeta d x d t \\
\leq & \left(\int_{\rho^{\theta}}^{2 \rho^{\theta}} \int_{\rho^{2} \leq|x|^{2} \leq 2 \rho^{2}}|x|^{\alpha} u^{p} \zeta\left\|\beta^{\frac{1}{q}}(x) u\right\|_{q}^{r} d x d t\right)^{\frac{1}{\alpha_{1}}} \\
& \times\left(\int_{\rho^{\theta}}^{2 \rho^{\theta}}\left(\int_{\rho^{2} \leq|x|^{2} \leq 2 \rho^{2}} \beta^{-\frac{r \alpha_{2}}{q \alpha_{1}}}(x)|x|^{-\frac{\alpha \alpha_{2}}{\alpha_{1}}}\left|\zeta_{t}\right|^{\alpha_{2}} \zeta^{-\frac{\alpha_{2}}{\alpha_{1}}} d x\right)^{\frac{\alpha_{1}^{\prime}}{\alpha_{2}}} d t\right)^{\frac{1}{\alpha_{1}^{\prime}}} \\
& +\left(\int_{\rho^{\theta}}^{2 \rho^{\theta}} \int_{\rho^{2} \leq|x|^{2} \leq 2 \rho^{2}}|x|^{\alpha} u^{p} \zeta\left\|\beta^{\frac{1}{q}}(x) u\right\|_{q}^{r} d x d t\right)^{\frac{1}{\alpha_{3}}}
\end{aligned}
$$




$$
\begin{aligned}
& \times\left(\int_{\rho^{\theta}}^{2 \rho^{\theta}}\left(\int_{\rho^{2} \leq|x|^{2} \leq 2 \rho^{2}} \beta^{-\frac{r \alpha_{4}}{q \alpha_{3}}}(x)|x|^{-\frac{\alpha \alpha_{4}}{\alpha_{3}}}|\Delta \zeta|^{\alpha_{4}} \zeta^{-\frac{\alpha_{4}}{\alpha_{3}}} d x\right)^{\frac{\alpha_{3}^{\prime}}{\alpha_{4}}} d t\right)^{\frac{1}{\alpha_{3}^{\prime}}} \\
& +\left(\int_{\rho^{\theta}}^{2 \rho^{\theta}} \int_{\rho^{2} \leq|x|^{2} \leq 2 \rho^{2}}|x|^{\alpha} u^{p} \zeta\left\|\beta^{\frac{1}{q}}(x) u\right\|_{q}^{r} d x d t\right)^{\frac{1}{\alpha_{1}}} \\
& \times\left(\int_{\rho^{\theta}}^{2 \rho^{\theta}}\left(\int_{\rho^{2} \leq|x|^{2} \leq 2 \rho^{2}} \beta^{-\frac{r \alpha_{2}}{q \alpha_{1}}}(x)|x|^{\left(-\sigma-\frac{\alpha}{\alpha_{1}}\right) \alpha_{2}} \zeta^{\frac{\alpha_{2}}{\alpha_{1}^{\prime}}} d x\right)^{\frac{\alpha_{1}^{\prime}}{\alpha_{2}}} d t\right)^{\frac{1}{\alpha_{1}^{\prime}}} .
\end{aligned}
$$

But $\int_{0}^{\infty} \int_{R^{N}}|x|^{\alpha} u^{p} \zeta\left\|\beta^{\frac{1}{q}}(x) u\right\|_{q}^{r} d x d t<\infty$ implies that

$$
\lim _{\rho \rightarrow \infty} \int_{\rho^{\theta}}^{2 \rho^{\theta}} \int_{\rho^{2} \leq|x|^{2} \leq 2 \rho^{2}}|x|^{\alpha} u^{p} \zeta\left\|\beta^{\frac{1}{q}}(x) u\right\|_{q}^{r} d x d t=0 .
$$

This infers that

$$
\int_{0}^{\infty} \int_{R^{N}}|x|^{\alpha} u^{p} \zeta\left\|\beta^{\frac{1}{q}}(x) u\right\|_{q}^{r} d x d t=0 .
$$

Now the assumption $\gamma \leq 0$ means

$$
N \leq \frac{2 q+\alpha q-r s}{q(p+r-m)-r}
$$

The proof of Theorem 1 is completed.

Next, we take the same test function in the proof of Theorem 1 and then Theorem 2 can be exactly proved as follows.

Proof of Theorem 2 Suppose that the assertion in Theorem 2 is not valid, and let $u \geq c>0$ be a nontrivial solution to problem (1.1), (1.3), we will show that this assumption leads to a contradiction. Assume that both $V(x)$ and $\beta(x)$ are singular at 0 . From (1.4) and (3.9), we get

$$
\int_{0}^{\infty} \int_{R^{N}}|x|^{\alpha} u^{p} \zeta\left\|\beta^{\frac{1}{q}}(x) u\right\|_{q}^{r} d x d t \leq C \rho^{\gamma}
$$

Combining this inequality with $u(x, t) \geq c>0$, we have

$$
\begin{aligned}
& \int_{0}^{\infty} \int_{R^{N}}|x|^{\alpha} u^{p} \zeta\left\|\beta^{\frac{1}{q}}(x) u\right\|_{q}^{r} d x d t \\
& \quad \geq C \rho^{\theta+\alpha+N+\frac{r}{q}(N-s)} \int_{0}^{2} \int_{|\xi| \leq 2}|\xi|^{\alpha} \zeta d \xi\left(\int_{|\xi| \leq 2}|\xi|^{-s} d \xi\right)^{\frac{r}{q}} d \tau \\
& \quad \geq C \rho^{\theta+\alpha+N+\frac{r}{q}(N-s)} .
\end{aligned}
$$

By (3.11)-(3.12), we have

$$
\gamma \geq \theta+\alpha+N+\frac{r}{q}(N-s)
$$


i.e.,

$$
s<N+\frac{q(2+\alpha)}{r} .
$$

The proof of Theorem 2 is completed.

Proof of Theorem 3 Following the proof of Theorem 1, we obtain the following estimate analogous to the estimate of Theorem 3:

$$
\begin{aligned}
& \int_{0}^{\rho^{\theta}} \int_{R^{N} \cap\{|x| \leq \sqrt{2} \rho\}}|x|^{\alpha} u^{p} \zeta\left\|\beta^{\frac{1}{q}}(x) u\right\|_{q}^{r} d x d t+\int_{R^{N} \cap\{|x| \leq \sqrt{2} \rho\}} u_{0}(x) \zeta(x, 0) d x \\
& +\int_{R^{N} \cap\{|x| \leq \sqrt{2} \rho\}} \omega(x) \zeta(x, 0) d x \leq C \rho^{\theta+\left(N-2 \alpha_{4}+\frac{r \alpha_{4}}{q \alpha_{3}}-\frac{\alpha \alpha_{4}}{\alpha_{3}}\right) \frac{\alpha_{3}^{\prime}}{\alpha_{4}}},
\end{aligned}
$$

whence

$$
C_{\omega} \rho^{\theta} \leq \int_{0}^{\rho^{\theta}} \int_{R^{N} \cap\{|x| \leq \sqrt{2} \rho\}} \omega(x) \zeta d x d t \leq C \rho^{\theta+\left(N-2 \alpha_{4}+\frac{r s \alpha_{4}}{q \alpha_{3}}-\frac{\alpha \alpha_{4}}{\alpha_{3}}\right) \frac{\alpha_{3}^{\prime}}{\alpha_{4}}}
$$

if $\rho$ is such that

$$
\int_{R^{N} \bigcap\{|x| \leq \sqrt{2} \rho\}} \omega(x) \zeta(x, 0) d x \geq c_{\omega} \equiv \text { const }>0 .
$$

Assuming that

$$
\gamma_{1}=\left(N-2 \alpha_{4}+\frac{r s \alpha_{4}}{q \alpha_{3}}-\frac{\alpha \alpha_{4}}{\alpha_{3}}\right) \frac{\alpha_{3}^{\prime}}{\alpha_{4}}<0,
$$

we obtain a contradiction by letting $\rho \rightarrow \infty$. Next, from (3.15), we obtain

$$
\begin{gathered}
q(p+r)(N-2)<m(\alpha q-r s)+m N(q+r) \\
\Leftrightarrow \quad N<\frac{2 q(p+r)+m(\alpha q-r s)}{q(p+r)-m(q+r)} .
\end{gathered}
$$

It must be noticed that if $N \leq 2$, then (3.16) is obviously fulfilled. The proof of Theorem 3 is completed.

\section{Acknowledgements}

The authors would like to deeply thank all the reviewers for their insightful and constructive comments.

Funding

The work of the second author (Fang) was supported by the Natural Science Foundation of Shandong Province of China (No. ZR2019MA072) and the Fundamental Research Funds for the Central Universities (No. 201964008).

\section{Abbreviations}

Not applicable.

Availability of data and materials

Data sharing is not applicable to this article as no data sets were generated or analyzed during the current study. 
Authors' contributions

All authors contributed equally and significantly in writing this article. All authors read and approved the final manuscript.

\section{Author details}

'School of Mathematical and Computer Science, Shanxi Normal University, Linfen, P.R. China. ${ }^{2}$ School of Mathematical

Sciences, Ocean University of China, Qingdao, P.R. China.

\section{Publisher's Note}

Springer Nature remains neutral with regard to jurisdictional claims in published maps and institutional affiliations.

Received: 26 August 2019 Accepted: 28 February 2020 Published online: 11 March 2020

\section{References}

1. Bebernes, J., Bressan, A.: Thermal behavior for a confined reactive gas. J. Differ. Equ. 44(1), 118-133 (1982)

2. Bebernes, J., Eberly, D.: Mathematical Problems from Combustion Theory. Springer, New York (1989)

3. Furter, J., Grinfield, M.: Local vs. nonlocal interactions in populations dynamics. J. Math. Biol. 27(1), 65-80 (1989)

4. Fujita, $H_{\text {.: }}$ On the blowing up solutions of the Cauchy problem for $u_{t}=\Delta u+u^{\alpha+1}$. J. Fac. Sci., Univ. Tokyo, Sect. I 13(2), 109-124 (1966)

5. Galaktionov, V.A., Vazquez, J.L.: Continuation of blowup solutions of nonlinear heat equations in several space dimensions. Commun. Pure Appl. Math. 50(1), 1-67 (1997)

6. Mitidieri, E., Pohozaev, S.I.: Nonexistence of positive solutions for quasilinear elliptic problems on $R^{N}$. Proc. Steklov Inst. Math. 227, 1-32 (1999)

7. Mitidieri, E., Pohozaev, S.I.: Nonexistence of weak solutions for some degenerate elliptic and parabolic problems on $R^{N}$. J. Evol. Equ. 1(2), 189-220 (2001)

8. Kartsatos, A.G., Kurta, V.V.: On the critical Fujita exponents for solutions of quasilinear parabolic inequalities. J. Math. Anal. Appl. 269(1), 73-86 (2002)

9. Jiang, Z.X., Zheng, S.N.: A Liouville-type theorem for a doubly degenerate parabolic inequality. Acta Math. Sci. (Chin Ser.) 30(3), 639-643 (2010)

10. Li, X.H., Li, F.Q.: A priori estimates for nonlinear differential inequalities and applications. J. Math. Anal. Appl. 378(2), 723-733 (2011)

11. Li, X.H., Li, F.Q.: Nonexistence of solutions for singular quasilinear differential inequalities with a gradient nonlinearity. Nonlinear Anal. 75(5), 2812-2822 (2012)

12. Wei, G.M.: Nonexistence of global solutions for evolutional p-Laplace inequalities with singular coefficients. Chin. Ann. Math., Ser. A 28(3), 387-394 (2007)

13. Fang, Z.B., Xu, L.J.: Liouville theorems for a singular parabolic differential inequality with a gradient term. J. Inequal. Appl. 2014, Article ID 62 (2014)

14. Yang, C.X., Zhao, L.Z., Zheng, S.N.: The critical Fujita exponent for the fast diffusion equation with potential. J. Math. Anal. Appl. 398, 879-885 (2013)

15. Liu, C.C.: The critical Fujita exponent for a diffusion equation with a potential term. Lith. Math. J. 54(2), 182-191 (2014)

16. Ishige, K.: On the Fujita exponent for a semilinear heat equation with a potential term. J. Math. Anal. Appl. 344, 231-237 (2008)

17. Pinsky, R.: The Fujita exponent for semilinear heat equations with quadratically decaying potential or in an exterior domain. J. Differ. Equ. 246(6), 2561-2576 (2009)

18. Chen, C.S., Huang, J.C.: Some nonexistence results for degenerate parabolic inequalities with local and nonlocal nonlinear terms. J. Nanjing Univ. Math. Biq. 21(1), 12-20 (2004)

19. Huang, J.C., Chen, C.S.: Nonexistence of global solutions to evolution problems with nonlocal sources. J. Nanjing Univ. Math. Biq. 21(2), 213-218 (2004)

\section{Submit your manuscript to a SpringerOpen ${ }^{\circ}$ journal and benefit from:}

- Convenient online submission

- Rigorous peer review

- Open access: articles freely available online

- High visibility within the field

Retaining the copyright to your article

Submit your next manuscript at $>$ springeropen.com 